\title{
La metafísica moderna y la teoría de la complejidad
}

Marcelino Rojas Carrero'. Recibido en Septiembre 2011, aprobado en Octubre 2011

\begin{abstract}
"El conocimiento aislado que ha tenido un grupo de especialistas en un campo estrecho no tiene en sí mismo valor de ninguna clase. Sólo tiene valor en el sistema teórico que lo reúne con todo el resto del conocimiento, y solamente en la medida en que contribuya realmente, en estas síntesis, a responder a la pregunta: ¿Qué somos nosotros?"
\end{abstract}

SCHRÖDINGER.

\section{Resumen}

Con el presente artículo se pretende contribuir al análisis y reflexión sobre la llamada crisis de la modernidad, en orden a plantear en qué consiste tal crisis, algunas insuficiencias fundamentales que padece y describir algunas de las reacciones que ha generado.

Palabras claves

Filosofía de la realidad, metafísica, teoría de la complejidad, metafísica moderna, filosofía moderna, postmodernidad.

\section{Abstract}

This article aims to contribute with analysis and reflection on the so called crisis of modernity, to define what this crisis is, what its reactions have been, and to outline some of its fundamental weaknesses.

Keywords:

Philosophy of reality, metaphysics, complexity theory, metaphysics of modernity, modern philosophy, postmodernity. 


\section{Introducción}

El fenómeno de la crisis de la modernidad sale a la luz en la crisis del proyecto de sociedad moderno, que se evidencia, según Nicolás ${ }^{2}$ en situaciones de injusticia social, económica, política y cultural para una gran parte de la humanidad; en el relativismo moral, en la pérdida de convicciones, en el hedonismo y el narcisismo exacerbado y generalizado; en cierta sensación de desfondamiento de la realidad; en nihilismos y escepticismos de todo tipo; en la fragmentación y dispersión del saber y de la vida, entre otros.

Ante tal crisis, existen una variedad de reacciones: continuistas, transformadoras y rupturistas. Sin embargo, ninguna constituye una auténtica superación de la crisis de la modernidad, precisamente porque fundan sus bases en la misma metafísica o teoría de la realidad.

En este artículo lo que se encuentra en cuestión no son los logros de la modernidad, ni de las reacciones de la modernidad, sino sus insuficiencias. Pero no en el nivel lógico ni en el ético ni en el político, sino en el nivel de la teoría de la realidad, entendido también como metafísica. El objetivo es trazar un esbozo del problema en cuestión y, además, de su posible solución.

\section{Metafísica y Filosofía de la Realidad}

Para comenzar es necesario superar la idea tradicional que se tiene de la metafísica. La metafísica ha sido entendida a lo largo de dos milenios como el estudio de lo trascendente, de lo que está más allá de la física. El concepto de metafísica surgió históricamente después de la muerte de Aristóteles, cuando sus tratados sobre la realidad fueron titulados por sus estudiosos con el término de metafísica.

Para Aristóteles el estudio de la realidad en tanto realidad era un tema de la Filosofía Primera. Esto es, el estudio de los principios generales de la realidad que era anterior a, y ponía las bases teóricas para la ética, la política, la lógica, entre otras.

Según González ${ }^{3}$, el objeto de estudio de la Filosofía Primera es la realidad, pero no en sus particularidades, sino en su totalidad. Este preguntarse ¿qué es la realidad en su totalidad? no es trascendente, sino trascendental. Esta diferencia es crucial para entender correctamente a la Filosofía Primera y el problema en torno a las metafísicas antiguas, modernas y contemporáneas.

2 J. Nicolás. Alternativas actuales a la crisis de la metafísica de la moderna. Granada: Ediciones de la Universidad de Granada, 2009

3 A. González. Introducción a la práctica de la filosofía. San Salvador: Ediciones UCA, 2000 
Lo anterior significa que el objeto de estudio de la Filosofía Primera no es lo trascendente, lo que está más allá de la física, sino lo trascendental, lo qué es la realidad en tanto totalidad. Es decir, cuáles son sus principios, cuál es su estructura, cuáles son sus caracteres universales y cuáles son sus tendencias.

En cambio, la pregunta por lo trascendental mira a aquello en que todo lo real coincide por el mero hecho de ser real. 0 sea, aquello que es común a toda la realidad. Es decir, se deja de lado lo particular para atender a lo general.

En conclusión, por alrededor de dos milenios ha habido un extravío de la teoría de la realidad causado por el dualismo y por el idealismo, causas que en este artículo únicamente se mencionan de pasada- y se deja para futuros artículos-, pero que alcanza hasta el siglo XX y que permanece con cierta actualidad sociocultural hasta el siglo XXI.

Por el momento, hasta aquí lo que tiene que ver con el objeto de estudio de la Filosofía Primera, esperando que haya quedado suficientemente claro las diferencias en torno a trascendente y lo trascendental, crucial para comprender la diferencia entre Metafísica y Filosofía Primera.

\section{La Metafísica y la Filosofía Moderna}

La filosofía moderna se caracteriza por una confianza absoluta en el poder de la razón. Esto es, lo que Nicolás llama la modernidad ilustrada. 0 dicho de otra manera, la filosofía moderna sienta sus bases en las ideas que los ilustrados tenían acerca de la realidad.

Según Nicolás, la ilustración es un movimiento intelectual y sociopolítico complejo, y una de sus características propias es el ansia de libertad en los ámbitos teóricos y prácticos. En esta primera y simple idea se deja translucir el signo del dualismo entre teoría y práctica que caracteriza a la metafísica moderna. La realidad es dividida intelectualmente en teoría y práctica.

Este dualismo en la realidad es la columna vertebral no sólo de la modernidad, sino también de la edad media y una parte significativa de la edad antigua. Este hecho no permitirá tener un acceso a la realidad en tanto realidad, sino en la creación de teorías-metafísicas que se alejaron de la realidad de las cosas mismas (Husserl).

Durante cuatro siglos el programa de la modernidad ha sido la racionalización de la realidad, con la voluntad puesta en aliviar y mejorar el estado de la humanidad. Esto siguiendo el ideal proclamado por Bacón. Este proyecto se concretó en un extraordinario desarrollo científico y en la teoría política del 
liberalismo. Surgió de esta manera una concepción científica de la racionalidad que llega hasta nuestros días en las corrientes empiristas y críticas.

Como se puede notar se trata de una reducción del saber a lo demostrable, ya sea empírica o lógicamente. Lo más grave es que se redujo la realidad al saber científico (lo demostrado) y se ha tenido por realidad el producto de la razón, tratando como igual lo que es diferente. De ahí que la realidad se entendió como un proceso lineal de progreso infinito, causal y determinado.

Esta reducción y homogenización de la realidad realizada por la filosofía moderna llevó a tratar con el mismo método a objetos de estudio diferentes. Por ejemplo, durante siglos se ha pregonado la idea de que lo más propio y específico de la racionalidad científica es el método. Y no cualquier método, sino el método científico aplicado en las ciencias naturales. De esta manera se negó la cientificidad a los demás tipos de saberes, y, además, se le negó el derecho a otros métodos de acceso a la realidad. Precisamente porque la única vía de acceso a la realidad le corresponde al método científico. Claro, se trata de la versión reducida e idealizada de la racionalidad y de la realidad. 0 lo que es igual, la versión moderna de la realidad o metafísica moderna.

Un ejemplo paradigmático de este error de la metafísica moderna es el intento del positivismo lógico de eliminar la metafísica misma. El positivismo lógico realizó un intento de aniquilación de la metafísica, y sin darse cuenta comenzó a destruir los pilotes sobre los que se sostenía su propia metafísica y filosofía. El intento del positivismo lógico ha sido fuertemente rebatido, entre otros, por el racionalismo crítico de Karl Popper ${ }^{4}$. Sin embargo, el mismo racionalismo crítico de Karl Popper permaneció bajo el paradigma de la metafísica moderna. Esta vez bajo la versión de la confianza en el poder crítico de la razón.

Otro grupo de pensadores que han criticado fuertemente a la metafísica moderna son los llamados filósofos postmodernos. Estos se expondrán más adelante como el intento de una ruptura con la modernidad.

\section{3. ¿En qué consiste la crisis de la filosofía y de la metafísica de la modernidad?}

La cultura europea moderna a partir del siglo XVIII se puede caracterizar por la explotación de la razón, el criticismo, la secularización del pensamiento, la búsqueda de la autonomía y de la libertad. Esta orientación racionalista ilustrada conocida como la modernidad es la que se encuentra en crisis.

Según Nicolás las causas de la crisis de la modernidad ilustrada están cargadas de cierta experiencia de desilusión ante las promesas de "aliviar y mejorar el estado de la humanidad". Y las críticas se concentran alrededor de la idea que se tiene de razón y de su configuración científico-técnica, frente a 
otras concepciones de la razón que se dejaron en segundo plano- las ideas de realidad, la idea de progreso al infinito, las ideas políticas de igualdad, libertad y fraternidad, entre otras.

Sin embargo, la racionalidad moderna simplista, reductora y disyuntiva de la realidad ha demostrado su insuficiencia en la ética y en la política: "ser capaz de aliviar y mejorar el estado de la humanidad". O dicho de otra manera, la racionalidad moderna no es suficiente para hacer una vida humana buena. Un ejemplo emblemático de la insuficiencia de la metafísica moderna -en que se fundamenta su racionalidad, su quehacer científico, ético y político- es que sacrifica la diversidad, también real, en aras de la unidad, simplificando, reduciendo y oponiendo unos elementos de la realidad frente a otros. De esta manera la metafísica moderna se vuelve ciega a la diversidad en la unidad de la realidad misma.

Por lo tanto, era un resultado coherente que esta teoría de la realidad moderna entrara en crisis: precisamente porque se no corresponde con la realidad misma. O lo que es igual, en la metafísica moderna existe una inadecuación entre teoría y realidad que es necesario superar.

\section{Reacciones ante la crisis de la filosofía y metafísica moderna}

Ante la crisis de la filosofía y de la metafísica moderna se ubican dos tipos de reacciones: la primera, aquella que defiende el fin del proyecto postmoderno con sus ideas acerca de la razón, de la realidad, de la libertad, de la ciencia y de la política. La segunda, aquella que defiende la modernidad y propone profundizarla.

En el caso de la primera reacción se trata en definitiva del fin de un modelo de racionalidad, de un modelo de realidad y de un proyecto ético-político. En el caso de la segunda reacción se trata de la continuidad por profundización de la modernidad.

Según Nicolás los filósofos llamados postmodernos constituyen un intento de ruptura con la modernidad, en cambio el racionalismo crítico y el naturalismo se ubican en la línea de continuidad por profundización y radicalización de la modernidad.

El racionalismo crítico es un intento de continuar con una racionalidad científicatécnica-industrial. Según los racionalistas críticos si la racionalidad moderna ha fracasado es por falta de radicalidad. Es necesario impulsar la crítica racional ahí donde existen resistencias ${ }^{5}$. El racionalismo crítico rechaza toda 
fundamentación del saber y propone en su lugar el principio del examen crítico. A su vez mantiene la idea moderna de la unificación del método científico para todos los esfuerzos de investigación en todos los campos del saber.

Si bien es cierto, en el racionalismo crítico se rompe con el dogmatismo en la ciencia. Sin embargo, mantiene la idea de una sola vía de acceso a la realidad: el método científico. Como se puede notar se continúa con la idea de ciencia de la modernidad: un saber únicamente es verdadero si se sigue el método científico. Esto obliga a pensar en y a mantener un proyecto de ciencia unificada, sin reconocer en la realidad la diversidad de objetos de estudio, y por lo tanto la diversidad de vías de acceso a la realidad.

Por otra parte, el naturalismo conserva la confianza en que la razón es el instrumento más adecuado para conseguir el progreso humano. Otras ideas de la modernidad que mantiene el naturalismo son: la ciencia empírica es la expresión privilegiada de la racionalidad, la problematicidad del mundo externo, y fiabilidad de la sensibilidad. Los dos puntos de vista privilegiados de la naturalización de la razón son los de la psicología y de la biología, pero siguiendo el método científico. Se trata de un intento por explicar todo el ámbito racional desde el punto de vista psicológico y biológico. De tal manera, que se continua asistiendo al mismo intento de reducir la realidad, en este caso la humana, a lo biológico y/o psicológico.

Un intento de ruptura la constituye la crítica de los llamados postmodernos. Entre ellos destacan F. Lyotard y G. Vattimo. Para estos la metafísica científicotécnica moderna debe ser superada. Según los postmodernos, la filosofía moderna ha configurado la historia y la experiencia de los últimos cuatro siglos. El pensamiento postmoderno se caracteriza por una ruptura con las ideas de fundamentación del saber y la universalidad de las teorías y de la verdad.

\section{Crítica de los filósofos Postmodernos a la filosofía y metafísica de la modernidad}

Según Nicolás la crítica llamada postmoderna consiste en el abandono de la lógica de la identidad por la lógica de la diferencia, en el fin de los grandes relatos y del sujeto sustancial, en la prioridad de lo individual frente a la totalidad, de la corporalidad, del sentimiento, de la voluntad, de la sensibilidad, del momento presente sobre el futuro; de lo contingente y de la moda frente a lo permanente, lo estable y lo duradero. Los postmodernos sustituyen el intento de fundamentación (epistemología) por la interpretación del saber (hermenéutica), y el deber (lo ético) por el cuidado de sí (lo estético).

De entrada se nota que el intento de ruptura de los postmodernos es insuficiente, constituyen una crítica a la filosofía moderna, pero no una superación de 
la metafísica moderna. La filosofía postmoderna, al igual que la moderna, continúa bajo el paradigma de la simplicidad, es reduccionista y disyuntora de la realidad. Es la versión opuesta de lo mismo: la filosofía moderna reduce lo distinto en la identidad, en cambio la filosofía postmoderna reduce la identidad en lo distinto, traicionando a la realidad en tanto realidad, que es una en la diversidad y diversa en la unidad. De ahí que en el proyecto ético y político de la modernidad se sacrifique a lo distinto $\mathrm{y} / \mathrm{o}$ a la diversidad en aras de la unidad y/o de la identidad; $y$ en el proyecto ético y político de la postmodernidad -implícito en su crítica- se sacrifica la unidad y la identidad en aras de lo distinto y de lo diverso.

De lo anterior, se sigue que el intento de ruptura de los filósofos llamados postmodernos con la modernidad es un intento fallido, porque mantienen los mismos fundamentos metafísicos de simplicidad, reducción y disyunción de la realidad. La llamada "postmodernidad" constituye una crítica, pero no una superación de la modernidad. Por ende, el nombre de postmodernos es, estrictamente hablando, inadecuado porque precisamente no se encuentran más allá de la modernidad, sino bajo el mismo paradigma metafísico de la modernidad. 0 dicho de otra manera, comparten en el fondo la misma teoría de la realidad.

\section{Otros intentos de respuesta a la crisis de la filosofía y metafísica moderna: los reformistas}

Un tercer grupo de respuesta a la crisis de la modernidad la constituye la reformista. Esta propugna cambios desde dentro, sin renunciar a la herencia esencial de la modernidad. Según Nicolás, se pueden distinguir dos grupos reformistas: el neoconservador y el crítico. Entre los autores neoconservadores se ubican D. Bell y P. Berger, y entre los autores críticos, a J. Habermas, K. Apel y A. Wellmer.

Según la reacción neoconservadora la causa de la crisis de la modernidad no se encuentra en su origen, sino en una desviación de la moral moderna. Por lo que, proponen continuar con el proyecto moderno de progreso científicotecnológico y retornar a los valores morales de la economía capitalista y de la política liberal.

La propuesta reformista de los críticos a la crisis de la modernidad consiste en un re-orientar la modernidad. El proyecto moderno se ha visto reducido a la racionalidad instrumental dejando de lado la racionalidad comunicativa y la racionalidad emancipadora. La ciencia se ha realizado al margen de la ética y de la estética. 0 dicho de otra manera, el progreso científico-tecnológico no ha sido seguido de un progreso ético y estético. 0 lo que es todavía peor, existe un 
dominio de la razón estratégica o instrumental sobre todos los demás ámbitos del saber y del obrar humano en sociedad.

Según Habermas ${ }^{6}$, el equilibrio entre ciencia, ética y estética ha de ser recompuesto desde el mundo de la vida a través de la acción comunicativa. Se trata de crear instituciones que liberen a los demás ámbitos del pensar y actuar humano del yugo de la racionalidad instrumental.

En definitiva, ambas reacciones reformistas propugnan una continuidad del proyecto moderno, pero corregido. Se trata de versiones recargadas de la modernidad.

\section{La teoría de la complejidad expuesta de acuerdo a Edgar Morin}

Le busquemos por donde le busquemos la filosofía de la modernidad, y las reacciones a la crisis de la modernidad que se han expuesto, se fundamentan en la misma teoría de la realidad: disyuntiva, simplista y reductora. Por ejemplo, la filosofía moderna, al igual que las reacciones filosóficas expuestas, son incapaces de asumir en su teoría de la realidad, que la realidad en tanto realidad sea orden y desorden a la vez (siguiendo el principio de no contradicción de la lógica formal). O lo que es todavía más profundo, que el orden procede de, se nutra de y tienda hacia el desorden.

Sin embargo, desde el punto de vista de la realidad en tanto realidad, y no desde el punto de vista de la teoría (ideas sobre), la física actual, en cuanto ciencia, admite la co-existencia de lo contradictorio en la realidad, y lo que es todavía más fuerte la interrelación dialéctica, recursiva y poiética entre el orden y el desorden. Esto es, una interrelación entre lo contradictorio -no permitida en la lógica formal-. O expresado en lenguaje ontológico, existe un diálogo recursivo y poiético entre el ser y el no ser.

Otro ejemplo paradigmático de la insuficiencia de la filosofía y de la metafísica moderna, es la existencia en la realidad en tanto realidad, de la complementariedad en el antagonismo de lo diferente, o dicho al revés, del antagonismo en la complementariedad de lo diferente. Según Morin ${ }^{7}$ se trata de un más allá del holismo y del reduccionismo: esto es, de un circuito relacional sistémico de lo diferente.

Afirmaciones de la teoría de la complejidad como el todo no es el todo, el todo es más que el todo, el todo es menos que el todo, el todo es más que las partes y a la vez menos que las partes, o la inversa las partes son menos que el todo y,

6 J. Habermas. Teoría de la acción comunicativa. Madrid: Editorial Taurus, 1999

7 E. Morin. La naturaleza de la naturaleza. París: Editorial Seuil, 1977 
a la vez, más que el todo, no tienen cabida en la racionalidad moderna.

Sin embargo, en la realidad en tanto realidad son verdaderas tales afirmaciones. 0 dicho metafóricamente: son verdaderas no porque lo dice la razón (lo racional), sino porque lo dice la realidad en tanto realidad (lo real).

La teoría de la complejidad ante la definición simplista, reductiva y disyuntora de la realidad, propia de la metafísica moderna, propugna una realidad compleja, que es interrelación y articulación, por ejemplo de lo distinto en la unidad, y viceversa, en un circuito relacional, recursivo y poiético.

La metafísica moderna ha llevado a la humanidad a un callejón sin salida. Siendo coherentes, para un moderno sólo queda la escuela del duelo: un saber global e integrado es imposible, sólo se puede conocer aislando, no es posible articular las ciencias del hombre a las ciencias de la naturaleza o viceversa. 0 dicho de otra manera, sólo podemos conocer un fragmento de la realidad y es imposible comunicar y articular esa multiplicidad de conocimientos con la vida.

De esta manera, la metafísica moderna ha desembocado en una única vía de acceso a la realidad, llamado el método científico, que procede aislando, separando, desuniendo, reduciendo a la unidad, midiendo. Y, según su criterio, a este método se deben los grandes descubrimientos científicos que tanto han servido a la humanidad.

Sin embargo, según Morin, estos logros se han pagado con una descomposición y atomización generalizada de los seres y de la cosas, con la disyunción e incomunicación entre lo distinto, con una parcelación absurda de la realidad y del saber, con la dislocación en mil saberes ignorantes. En consecuencia, la ciencia se ha vuelto incapaz de concebirse como praxis social, de controlar su poder de manipulación, y lo que es más grave, de controlar su manipulación por los poderes.

Para Morin la humanidad se encuentra con una ciencia sin sujeto, y con un objeto de la ciencia dislocado y desmigajado en mil y una disciplinas. Tal situación es una noche sobre el conocimiento que se debe superar. 0 dicho de otra manera, se trata de una separación entre la ciencia y la vida que urge superar.

Para Morin la disyunción y la simplificación se encuentran muertas en la base misma de la realidad física. Por ejemplo, cita el autor, siguiendo a la física actual, "la partícula subatómica no volverá a ser ya el elemento aislable, simple e indivisible". Y agrega, "confusión e incertidumbre no son las palabras últimas del saber, sino los signos precursores de la complejidad". 
Ahí donde la filosofía llamada moderna se agotó -la confusión y la incertidumbre-, es el punto de partida de la teoría de la complejidad: precisamente porque la metafísica moderna hundía sus raíces en una teoría de la realidad errónea, que simplificó, redujo y disgregó la realidad en mil y un pedazos de un rompecabezas, incapaz de volver a armar.

En conclusión, la teoría de la complejidad no es una reforma de la modernidad, sino el comienzo de una nueva teoría de la realidad. O dicho de otra manera, de una nueva filosofía de la realidad o filosofía primera, necesaria, para orientar el quehacer ético y político de la humanidad. Y siendo todavía más preciso, es una superación, no de la crisis de la metafísica moderna, sino de la metafísica misma de la modernidad.

\section{Conclusiones}

La filosofía moderna se encuentra en un estado crisis a causa de una metafísica o teoría de la realidad que es necesario superar: precisamente porque no se corresponde con la realidad en tanto realidad. La crisis de la modernidad es la crisis de un modelo de racionalidad, de un modelo de realidad y de un proyecto ético-político.

Ante tal crisis, le han sucedido tres tipos de reacciones: la continuista por profundización, la transformadora crítica o re-orientadora, y la rupturista.

Sin embargo, ninguna de las tres reacciones constituyen una superación de la crisis de la modernidad, precisamente porque comparten las mismas ideas trascendentales acerca de la realidad (metafísica): atomizada, simple, disyuntiva y reductiva.

El intento de superar la crisis de la modernidad ha conducido a un callejón sin salida, precisamente porque sus ideas acerca de la realidad son inadecuadas a la realidad en tanto realidad.

La teoría de la complejidad constituye una auténtica superación, no de la crisis de la modernidad, sino de la metafísica misma de la modernidad -que es la causa de la crisis de la modernidad-, precisamente porque aborda a la realidad desde una filosofía primera distinta: se trata de la realidad en tanto realidad compleja, que es interrelación y articulación, en un circuito interactivo, recursivo y poiético.

En conclusión, la principal tarea actual de la filosofía se ubica no el terreno lógico ni en el ético-político, sino en el de la filosofía primera o teoría de la realidad, sobre la cual se funda la lógica, la ética y la política. 


\section{Bibliografía}

H. Albert. Tratado sobre la razón crítica. Buenos Aires: Editorial Sur, 1973

A. González. Introducción a la práctica de la filosofía. San Salvador: Ediciones UCA, 2000

J. Habermas. Teoría de la acción comunicativa. Madrid: Editorial Taurus, 1999

E. Morin. La naturaleza de la naturaleza. París: Editorial Seuil, 1977

J. Nicolás. "Alternativas actuales a la crisis de la metafísica de la moderna". Granada: Ediciones de la Universidad de Granada, 2009

K. Popper. La lógica de la investigación científica. Madrid: Editorial Tecnos, 1935 